\title{
The diagnostic utility of the anti-CCP antibody test is no better than rheumatoid factor in South Africans with early rheumatoid arthritis
}

\author{
Bridget Hodkinson • Pieter W. A. Meyer • Eustasius Musenge $\cdot$ Mahmood M. T. Ally • \\ Ahmed A. Wadee • Ronald Anderson • Mohammed Tikly
}

Received: 3 September 2009 /Revised: 29 November 2009 / Accepted: 4 January 2010

(C) Clinical Rheumatology 2010

\begin{abstract}
To establish the diagnostic utility of the anticyclic-citrullinated peptide antibody $(\mathrm{aCCP})$ test in Black South Africans with early rheumatoid arthritis (RA). A cross-sectional study comparing the rheumatoid factor (RF) and aCCP status in RA patients and a control group consisting of healthy subjects, and patients with systemic lupus erythematosus (SLE) and scleroderma. The sensitivity, specificity, positive (PPV) and negative predictive values of the aCCP test alone were $82.5 \%, 84.9 \%, 87.6 \%$ and $79 \%$ versus $81.7 \%, 90.7 \%, 92.5 \%$ and $78 \%$ for RF alone. The best specificity (95.3) and PPV (95.8\%) was observed when both
\end{abstract}

B. Hodkinson $\cdot$ M. Tikly $(\square)$

Division of Rheumatology, Chris Hani Baragwanath Hospital and University of the Witwatersrand,

P.O. Bertsham,

Johannesburg, South Africa

e-mail: tikly.mohammed01@gmail.com

P. W. A. Meyer $\cdot$ R. Anderson

Department of Immunology, Medical Research Council

Unit for Inflammation and Immunity, Faculty of Health Sciences,

University of Pretoria and Tshwane Academic Division of the

National Health Laboratory Service,

Pretoria, South Africa

E. Musenge

Epidemiology Centre, School of Public Health,

University of the Witwatersrand,

Johannesburg, South Africa

M. M. T. Ally

Department Internal Medicine, Steve Biko Pretoria Academic

Hospital and University of Pretoria,

Pretoria, South Africa

A. A. Wadee

Division of Immunology, National Health Laboratory Services and University of the Witwatersrand,

Johannesburg, South Africa
$\mathrm{aCCP}$ and RF tests were positive. Patients with erosive disease had a significantly higher mean RF titre compared with those with non-erosive disease $(p=0.007)$. There was a trend towards an association of smoking $(\mathrm{OR}=4.1,95 \%$ $\mathrm{CI}=0.9-18.6)$ and functional disability $(p=0.07)$ with RF-positive status. No similar clinical associations were observed with aCCP. Almost a third of SLE patients were aCCP positive. Despite the best specificity and PPV observed when both the aCCP and RF tests were positive, our findings suggest that testing for aCCP is only cost-effective in the RF-negative patient in whom there is a strong clinical suspicion of RA.

Keywords Africa - Anti-CCP antibodies · Blacks · Rheumatoid arthritis $\cdot$ Rheumatoid factor

Early aggressive treatment with traditional and biologic disease-modifying antirheumatic drugs (DMARDs) to prevent joint destruction and disability is now accepted as best practice in rheumatoid arthritis (RA) [1]. With this drive to intervene early, comes a need to diagnose RA promptly and accurately. The American College of Rheumatology (ACR) classification criteria were derived from analysis of patients with established disease [2]. Not surprisingly, these criteria have poor diagnostic performance in early RA.

Rheumatoid factor (RF) has been the principal serological marker for RA until recently and is included in the ACR criteria. However, because of its low sensitivity, particularly early in the course of the disease, and its limited specificity, there has been much interest in identifying novel diagnostic biomarkers in RA. Autoantibodies directed against citrulline, an amino acid that results from post-translational modification of arginine in inflamed synovial membranes, have been shown to have a high 
specificity of $93-98 \%$ in early RA [3]. These antibodies to cyclic-citrullinated peptide (aCCP) are also of prognostic value in predicting disease severity, erosive damage and response to therapy $[4,5]$.

Most studies on the diagnostic utility of the aCCP test in RA have been done in industrialized countries on subjects of European ancestry [6], with only a few done in the developing world [7-10]. In the absence of any published studies on the prevalence and specificity of aCCP in RA in sub-Saharan Africa, we undertook a cross-sectional case-control study to establish the diagnostic utility of the RF and aCCP tests in urbanized Black South Africans with early RA. The Human Ethics Committee of the Faculty of Health Sciences, University of the Witwatersrand approved the study.

\section{Subjects and methods}

One hundred and twenty Black South African DMARDnaive RA patients, $\geq 18$ years at disease onset, and with disease duration of $<2$ years were studied. All patients fulfilled the revised ACR classification criteria for RA and were attending the Arthritis Clinics at either of two tertiary hospitals in South Africa. These patients are participants in an ongoing observational study, the Gauteng Rheumatoid Evaluation Assessment Trial (GREAT) study.

Disease activity was assessed using the 28 joint disease activity score (DAS28) [11]. The modified Health Assessment Questionnaire (mHAQ) was used to assess functional disability [12]. The presence of nodulosis and a smoking history were also documented. Patients were classified as having erosive disease based on baseline X-rays of the hands and feet. The control group consisted of healthy subjects $(n=30)$, systemic lupus erythematosus (SLE) patients $(n=35)$ and systemic sclerosis (SSc) patients $(n=28)$. In the SLE group, the presence of active tuberculosis (TB) was also noted because of recent reports of an association between aCCP and TB [13, 14], coupled with the frequent occurrence of TB in our SLE patients [15].

Rheumatoid Factor (composite IgM, IgG, IgA) was assayed by nephelometry (Siemens Healthcare Diagnostics, BN Prospec Nephelometer, Newark, USA). aCCP were measured using a second-generation immunofluorimetric assay with the Immunocap 250 system and reagents and controls provided by the manufacturer (Phadia AB, Uppsala, Sweden). RF and aCCP were considered positive when the concentrations were greater than $15 \mathrm{IU} / \mathrm{ml}$ and $10 \mathrm{U} / \mathrm{ml}$, respectively.

Statistical analysis

Statistical Analysis was performed using STATA statistical software. The sensitivity, specificity and predictive powers, as well as Cohen's kappa coefficient for agreement were calculated for RF and aCCP tests. In the RA group, logistic regression was applied to test for clinical associations with aCCP and RF. A $p$ value $<0.05$ was considered significant.

\section{Results}

The majority of our patients ( $83 \%$ ) were female, $20 \%$ were smokers, and a fifth had rheumatoid nodules (Table 1). The overall group had very active disease as reflected by a mean DAS28 of 6.2 and mean swollen joint count (SJC) of 9.3, with moderate to severe functional disability with a mean mHAQ-disability index (mHAQ-DI) of 1.7, and almost half the patients had erosive disease.

The aCCP and RF tests performed similarly with respect to sensitivity, specificity and predictive powers (Table 2). The presence of either RF or aCCP had the highest sensitivity $(88.3 \%)$ and the best negative predictive value (84.3\%), while the presence of both RF and aCCP was highly specific for RA (95.3\%) and had the best positive predictive value $(95.8 \%)$. Of the $22 \mathrm{RA}$ patients who were RF negative, 8 (36.4\%) were aCCP positive. Cohen's kappa coefficient for agreement between RF and aCCP was moderate, but significant at $0.58(95 \% \mathrm{CI}=0.4-0.8)$.

Within the RA group, the presence of aCCP was not associated with any specific disease characteristics, including cigarette smoking, age of disease onset, gender, disease duration, disease activity, functional disability, nodulosis or erosive disease. The mean $( \pm \mathrm{SD}) \mathrm{RF}$ titre was significantly higher in patients with erosive disease compared to those with non-erosive disease $(704.4 \pm 1043.4 \mathrm{IU} / 1$ vs. $308.8 \pm$

Table 1 Demographic and clinical characteristics of South Africans with early rheumatoid arthritis $(n=120)$

\begin{tabular}{lc} 
Female (\%) & $100(83.3)$ \\
Age at onset in years, mean (SD) & $47.6(12.5)$ \\
Duration of disease (months), mean (SD) & $11.9(7.2)$ \\
Post menopausal females (\%) & $42(35.0)$ \\
Cigarette smokers (\%) & $24(20)$ \\
DAS28, mean (SD) & $6.2(1.3)$ \\
Swollen joint count, mean (SD) & $9.3(6.2)$ \\
Tender joint count, mean (SD) & $12.9(7.6)$ \\
Patient global assessment, mean (SD) & $64.8(2.2)$ \\
CRP, mean (SD) & $25.0(31.8)$ \\
mHAQ-DI, mean (SD) & $1.7(0.2)$ \\
Nodulosis (\%) & $22(18.3)$ \\
Erosive disease (\%) & $52 / 113(46.0)$ \\
\hline
\end{tabular}

$D A S 2828$ joint disease activity score, $m H A Q-D I$ modified health assessment questionnaire disability index 
Table 2 Diagnostic properties of anti-CCP antibody and rheumatoid factor tests in South Africans with early rheumatoid arthritis

\begin{tabular}{lccccc}
\hline & RA $(n=120)$ & Controls $(n=93)$ & Sensitivity \% & Specificity \% & PPV \% \\
\hline Anti-CCP & 99 & 14 & 82.5 & 84.9 & 87.6 \\
RF & 98 & $8 / 86$ & 81.7 & 90.7 & 79.0 \\
RF or anti-CCP & 106 & 18 & 88.3 & 80.6 & 78.0 \\
RF and anti-CCP & 91 & $4 / 86$ & 75.8 & 95.3 & 85.4 \\
\hline
\end{tabular}

$R F$ rheumatoid factor, anti-CCP anti-cyclic citrullinated peptide antibodies, $R A$ rheumatoid arthritis, $P P V$ positive predictive value, $N P V$ negative predictive value

405.2 IU/1, $p=0.007)$. A trend towards an association of RF positivity with cigarette smoking (OR 4.1, 95\% CI $=0.9$ 18.6, $p=0.06$ ) was observed and patients who were RF positive had higher mHAQ-DI (mean $=2.0 \pm 1.5$ vs. $1.7 \pm 0.7$, $p=0.07$ ).

As shown in Fig. 1, of the 14 control subjects who were aCCP positive, one was a healthy subject with a high titre of $952 \mathrm{U} / \mathrm{ml}, 2$ had SSc (one with a low titre and one with a high titre of $890 \mathrm{U} / \mathrm{ml}$ ) and 11 had SLE, ten of whom had low titres $($ mean $=15.9)$ and one with a high titre of $773 \mathrm{U} / \mathrm{ml}$. Analysis of the SLE group showed no significant association of aCCP with inflammatory arthritis (3/11 in aCCP-positive group vs. $8 / 24$ in aCCP-negative group) and active TB (1/11 in aCCP positive group vs. $2 / 24$ in aCCP-negative groups). Neither of the two aCCP-positive SSc patients had a history of inflammatory arthritis.

\section{Discussion}

In this study of early RA, the first to our knowledge in subSaharan Africans, the overall performance of the aCCP and $\mathrm{RF}$ tests in the diagnosis of RA was similar, with the RF test performing better in predicting erosive disease and functional disability. Both the tests showed good sensitivity and positive

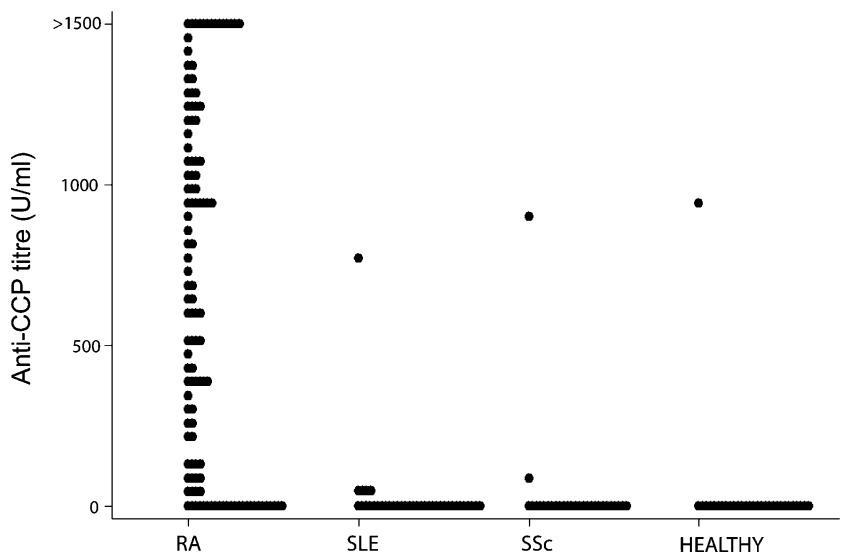

Fig. 1 Anti-CCP antibody titres in RA and control groups $(C C P$ cyclic citrullinated peptide, $R A$ rheumatoid arthritis, $S L E$ systemic lupus erythematosus, $S S c$ systemic sclerosis) predictive value, comparable to that reported in other populations [16]. While the specificity of the RF test is similar to studies of early RA in other populations, the specificity of the aCCP test was somewhat lower, primarily because of the high false positive rate in the SLE control group.

The combination of both RF and aCCP positivity had the highest specificity, indicating that, ideally, both tests be done for diagnostic certainty. In spite of this and given that, over a third of the RF-negative patients were aCCP positive, in a resource-limited setting such as ours and most of sub-Saharan Africa, testing simultaneously for both autoantibodies appears to carry little advantage over the RF test alone in the diagnosis of RA. Thus, a "stepwise" approach proposed previously by Nell et al. to autoantibody testing in a patient with inflammatory polyarthritis, starting with the RF test followed by the aCCP test only where the $\mathrm{RF}$ test is negative, is prudent [17].

Almost a third of the SLE group was aCCP positive, albeit in low titres in all except one patient. Although most older studies have shown aCCP to be distinctly uncommon in SLE patients [18], a very recent study of 159 Chinese SLE patients showed $27.3 \%$ of patients had aCCP, with a significantly higher prevalence in patients with inflammatory arthritis (42\%) compared with those without inflammatory arthritis (5.5\%) [19]. A similar association with inflammatory arthritis has also been described in African American SLE patients [20]. In the present study, we were unable to confirm this association, probably because of the small sample size.

The high false positive rate for aCCP in the SLE group might also be on the basis of non-specific increases in globulins in people of African origin as found previously with respect to antiphospholipid antibodies in SLE [21]. In light of the high occurrence of TB in our SLE patients (13), a further possible explanation is that some of these patients may be harbouring latent TB. The $5.6 \%$ frequency of aCCP in the SSc patients is similar to the $5-15 \%$ described previously in SSc [22, 23], but again we found no association with inflammatory arthritis as others have suggested.

The majority of patients in this cohort had very active disease with associated moderate to severe disability. This is consistent with our previous published findings in established RA in this population [24, 25]. Severe disease 
at presentation, at least in part, is a reflection of delay in referral for tertiary care. It underscores the need for improved clinical skills at lower levels of health care in recognizing milder forms of early inflammatory arthritis, and thus facilitates more effective intervention with relatively inexpensive drugs like methotrexate in the indigent RA patient in South Africa.

Since majority of patients in the present study had very active disease, the diagnostic utility of aCCP in patients with milder disease, commonly seen in most industrialized countries, remains unanswered. Another limitation is the relatively small sample size, which might explain why we failed to show a relationship between aCCP and smoking that has been reported previously [26]. We also could not assess the prognostic value of the aCCP test with respect to response to DMARDs and radiographic progression in this baseline cross-sectional analysis, but this is planned as part of the prospective longitudinal analysis of the GREAT study.

In summary, the RF test in a cohort of Black South Africans with early RA yields good specificity and sensitivity. Routine testing for aCCP adds little diagnostic advantage and testing for aCCP should be restricted to patients with inflammatory polyarthritis where the RF is negative.

\section{Disclosures None}

\section{References}

1. Landewe RB (2003) The benefits of early treatment in rheumatoid arthritis: confounding by indication, and the issue of timing. Arthritis Rheum 48:1-5

2. Arnett F, Edworthy SM, Bloch DA, McShane DJ, Fries JF, Cooper NS et al (1988) The American Rheumatism Association 1987 revised criteria for the classification. Arthritis Rheum 31:315-324

3. Niewold TB, Harrison MJ, Paget SA (2007) Anti-CCP antibody testing as a diagnostic and prognostic tool in rheumatoid arthritis. QJM 100:193-201

4. Kastbom A, Strandberg G, Lindroos A, Skogh T (2004) Anti-CCP antibody test predicts the disease course during 3 years in early rheumatoid arthritis (the Swedish TIRA project). Ann Rheum Dis 63:1085-1089

5. Machold KP, Stamm TA, Nell VP, Pflugbeil S, Aletaha D, Steiner $G$ et al (2007) Very recent onset rheumatoid arthritis: clinical and serological patient characteristics associated with radiographic progression over the first years of disease. Rheumatology (Oxford) 46:342-349

6. Nishimura K, Sugiyama D, Kogata Y, Tsuji G, Nakazawa T, Kawano S et al (2007) Meta-analysis: diagnostic accuracy of anticyclic citrullinated peptide antibody and rheumatoid factor for rheumatoid arthritis. Ann Intern Med 146:797-808

7. Abdel-Nasser AM, Mahmoud MH, El Mansoury TM, Osman AM (2008) Anti-CCP2 is an adjunct to, not a surrogate for, rheumatoid factor in the diagnosis of rheumatoid arthritis: diagnostic utility of anti-CCP2 antibodies in Egyptian patients with rheumatoid arthritis. Scand J Rheumatol 37:329-336

8. Aflaky E, Shenavandeh S, Ashraf MJ (2009) A comparison of performance of anti-cyclic citrullinated peptide 2 and citrullinated protein antibodies in the diagnosis of rheumatoid arthritis in Iranian patients. Rheumatol Int (in press)

9. Vanichapuntu M, Phuekfon P, Suwannalai P, Verasertniyom O, Nantiruj K, Janwityanujit S (2009) Are anti-citrulline autoantibodies better serum markers for rheumatoid arthritis than rheumatoid factor in Thai population? Rheumatol Int (in press)

10. Gupta R, Thabah MM, Aneja R, Kumar A, Varghese T, Chandrasenan PJ (2009) Usefulness of anti-CCP antibodies in rheumatic diseases in Indian patients. Indian J Med Sci 63:92-100

11. van der Heijde DM, van't Hof MA, van Riel PL, van de Putte L (1992) Disease activity score. Ann Rheum Dis 51:140

12. Kirwan JR, Reeback JS (1986) Stanford Health Assessment Questionnaire modified to assess disability in British patients with rheumatoid arthritis. Br J Rheumatol 25:206-209

13. Elkayam O, Segal R, Lidgi M, Caspi D (2006) Positive anti-cyclic citrullinated proteins and rheumatoid factor during active lung tuberculosis. Ann Rheum Dis 65:1110-1112

14. Kakumanu P, Yamagata H, Sobel ES, Reeves WH, Chan EK, Satoh M (2008) Patients with pulmonary tuberculosis are frequently positive for anti-cyclic citrullinated peptide antibodies, but their sera also react with unmodified arginine-containing peptide. Arthritis Rheum 58:1576-1581

15. Hodkinson B, Musenge E, Tikly M (2009) Osteoarticular tuberculosis in patients with systemic lupus erythematosus. QJM 102:321-328

16. Avouac J, Gossec L, Dougados M (2006) Diagnostic and predictive value of anti-cyclic citrullinated protein antibodies in rheumatoid arthritis: a systematic literature review. Ann Rheum Dis 65:845-851

17. Nell VP, Machold KP, Stamm TA, Eberl G, Heinzl H, Uffmann M et al (2005) Autoantibody profiling as early diagnostic and prognostic tool for rheumatoid arthritis. Ann Rheum Dis 64:1731-1736

18. Schellekens GA, Visser H, de Jong BA, van den Hoogen FH, Hazes JM, Breedveld FC et al (2000) The diagnostic properties of rheumatoid arthritis antibodies recognizing a cyclic citrullinated peptide. Arthritis Rheum 43:155-163

19. Qing YF, Zhang QB, Zhou JG, Yuan GH, Wei J, Xing Y et al (2009) The detecting and clinical value of anti-cyclic citrullinated peptide antibodies in patients with systemic lupus erythematosus. Lupus 18:713-717

20. Mikuls TR, Holers VM, Parrish L, Kuhn KA, Conn DL, Gilkeson G et al (2006) Anti-cyclic citrullinated peptide antibody and rheumatoid factor isotypes in African Americans with early rheumatoid arthritis. Arthritis Rheum 54:3057-3059

21. Gould T, Tikly M, Asherson R, Loizou S, Singh S (2006) Prevalence and clinical correlates of anti-phospholipid antibodies in South Africans with systemic lupus erythematosus. Scand J Rheumatol 35:29-34

22. Ingegnoli $\mathrm{F}$, Galbiati V, Zeni S, Meani L, Zahalkova L, Lubatti C et al (2007) Use of antibodies recognizing cyclic citrullinated peptide in the differential diagnosis of joint involvement in systemic sclerosis. Clin Rheumatol 26:510-514

23. Santiago M, Baron M, Miyachi K, Fritzler MJ, Abu-Hakima M, Leclercq $S$ et al (2008) A comparison of the frequency of antibodies to cyclic citrullinated peptides using a third generation anti-CCP assay (CCP3) in systemic sclerosis, primary biliary cirrhosis and rheumatoid arthritis. Clin Rheumatol 27:77-83

24. Tikly M, Zannettou N, Hopley M (2003) A longitudinal study of rheumatoid arthritis in South Africans. MedGenMed 5:2

25. Benitha R, Tikly M (2007) Functional disability and health-related quality of life in South Africans with rheumatoid arthritis and systemic lupus erythematosus. Clin Rheumatol 26:24-29

26. Klareskog L, Stolt P, Lundberg K, Kallberg H, Bengtsson C, Grunewald $J$ et al (2006) A new model for an etiology of rheumatoid arthritis: smoking may trigger HLA-DR (shared epitope)-restricted immune reactions to autoantigens modified by citrullination. Arthritis Rheum 54:38-46 